Euskal ikerketen aldizkaria | Revue d'études basques |

Revista de estudios vascos | Basque studies review

19 | 2016

Numéro XIX

\title{
Chenilles et coccinelles dans l'aire linguistique gasconne
}

\section{Rita Caprini}

\section{OpenEdition}

\section{Journals}

Édition électronique

URL : https://journals.openedition.org/lapurdum/3262

DOI : 10.4000/lapurdum.3262

ISSN : 1965-0655

\section{Éditeur}

IKER

Édition imprimée

Date de publication : 1 janvier 2016

Pagination : 171-175

ISBN : 978-2-95534-134-6

ISSN : $1273-3830$

Référence électronique

Rita Caprini, « Chenilles et coccinelles dans l'aire linguistique gasconne », Lapurdum [En ligne], 19|

2016, mis en ligne le 01 janvier 2021, consulté le 03 septembre 2021. URL : http://

journals.openedition.org/lapurdum/3262 ; DOI : https://doi.org/10.4000/lapurdum.3262 


\section{Chenilles et coccinelles dans l' aire linguistique gasconne}

Rita CAPRINI

Università di Genova

Depuis les temps de Gilliéron les chercheurs qui travaillent dans le domaine de la géographie linguistique savent très bien que la Gascogne présente, selon le point de vue de la dialectologie, des caractéristiques particulières qui ont attiré l'attention des savants.

En effet, quand l'idée des atlas régionaux français fut lancée (par Albert Dauzat en 1939, peu avant la guerre), la première aire dialectale de France qui s'ouvrit à la nouvelle exploration (aprés celle de Gilliéron et Edmont) fut la Gascogne, et l'Atlas Linguistique et ethnographique de la Gascogne fut le premier à paraître (Paris, CNRS, 1954), signé par Jean Séguy. En indiquant les raisons de son initiative, il écrivait dans l'Introduction: "Il m'avait paru nécessaire de reprendre, sur le plan régional, l'oeuvre de mon ancien maitre Gilliéron, qui si magistrale fut-elle, ne pouvait être le dernier mot de la dialectologie française." Il se plaignait de la disparition prochaine de nombreux patois. Aujourd'hui, soixante ans après, on sait bien que cela s'est bien produit.

Jean Séguy (1914-1973) fut titulaire de la chaire de linguistique romane à l'Université de Toulouse depuis 1950, et il portait un intérêt tout particulier pour les noms des plantes et des animaux, étant aussi un botaniste et un biologiste. Il se pencha surtout sur le problème des relations entre les catégories scientifiques et celles du savoir populaire, problème centrale pour l'étude du lexique dialectale. On sait bien que la classification scientifique des plantes et des animaux fut nécessaire en Europe à cause de l'extrême variété des noms populaires. On parle désormais depuis un demi-siècle d"'ethnoscience" comme domaine d'étude non seulement pour les ethnologues mais aussi pour les linguistes qui travaillent sur les dialectes d'Europe.

En travaillant pour l'Atlas Linguistique Roman depuis presque trente années, dédiant mon attention surtout aux noms d'animaux (j'ai signé les cartes sur les noms romans de la chenille, du rougegorge, déjà parues, et celle de la coccinelle, sous presse), j'ai constaté que cette partie du lexique nous révèle beaucoup de notre passé de croyances. Bien entendu, la langue conserve les traces des croyances trés éloignées dans le temps, dont souvent les locuteurs n'ont plus aucune connaissance consciente. En outre, j'ai constaté que, parmi les noms d'animaux, certains comme la chenille et la coccinelle présentent une très grande variété, tandis que d'autres, comme le rougegorge, pour ne citer que ceux que j'ai traité pour l'ALiR, sont plus simples et surtout ne révèlent pas l'arrière-plan presque mythique des autres. L'origine de cette différence demeure, en l'état actuel de nos connaissances, encore mystérieuse.

J'ai choisi alors, entre les cartes de l'Atlas Linguistique et ethnographique de la Gascogne, la carte 55 "chenille" e la carte 1223 "coccinelle" pour constater la présence ou l'absence des types romans plus fréquents.

De ce point de vue, la carte gasconne des noms de la chenille est un vrai condensé 
des noms romans de cette petite bête : je donne ici une liste des types, dans una transcription normalisée qui ne prend pas en compte les variétés phonétiques, qui ne font pas l'objet de notre présente recherche. Il faut encore souligner que la différence entre deux espèces de chenille, les chenilles velues comme la processionnaire du pin, et les chenilles glabres, type piéride du choux, n'est généralement pas relévée dans la carte (comme dans la carte de l'ALiR que j’ai réalisée).

Deux types romans bien connus pour "chenille" se partagent la plupart de l'aire de la Gascogne : les continuateurs de lat. ERUCA, avec la signification 'la chenille', et le type gatamino, c'est-à-dire le type CATTA suivi par un adjectif ou un nom. Ce dernier type se trouve seulement en Gascogne.

Les continuateurs de lat. ERUCA sont répandus surtout en Espagne, où ils dominent nettement sur les autres dénominations, mais ils se retrouvent aussi dans la partie centrale du domaine occitan, dans l'Italie septentrionale et centrale, en Sardaigne et en Corse. Les aires dont il est question ne forment pas une aire continue, et manquent absolument dans les parties extrêmes du domaine roman : le Portugal et la Romanie. Quant à la signification latine du terme, il faut noter que dès ses premières attestations (Virgile, Pline etc.), le terme désignait soit la chenille soit de différentes plantes alimentaires. Létymologie inconnue du mot nous empêche de donner des explications sûres : la chenille a peut-être pris le nom des plantes dont elle est gourmande, ou bien le zoonyme a subi un glissement de sens. Le mot latin a aussi des continuateurs romans parmi les phytonimes : il s'agit du type toscan rucola (une plante au parfum particulier, utilisée pour la préparation de différentes salades); de l'italien le mot est passé dans le fr. roquette (cf. a. it. rocchetta).

Du point de vue formel, les continuateurs directs d'ERUCA ne représentent pas la majorité, on trouve dans le domaine roman surtout des continuateurs irréguliers, par exemple dans le domaine occitan la consonne sourde de l'étymon latin se maintient souvent. Surtout dans une petite aire du domaine occitan sur les deux versants des Pyrénées, à cause du substrat basque, on a la conservation des sourdes intervocaliques latines: dans notre cas, dans l'aire plus orientale de l' Atlas Linguistique et ethnographique de la Gascogne, on trouve le type ruko avec une mauvaise coupure, comme dans les formes correspondants de l'aire italienne : la voyelle initiale du mot (e-, a-) est considérée partie de l'article, on passe alors de l'aruga à la ruga. La conservation de la sourde interne peut être causée par le contact avec le type kuko, fréquent dans l'aire gasconne, comme dans toute la Romania, pour désigner différentes petites bêtes. Le type kuko pour 'chenille' est enregistré par Seguy et ses collaborateurs dans un petit nombre de points situés entre l'aire de ruko et celle de gatamino.

A propos des continuateurs de lat. ERUCA en Gascogne il faut noter encore qu'un petit nombre de points d'enquête présentent des autres continuateurs irréguliers, un type à au- initial (aurugo, point 696), autres types avec des voyelles initiales inattendues comme u-, i- (points 680,690). Pour ces types romans on a pensé à une contamination avec lat. URO 'brûler'. Ce type se retrouve surtout dans le castillan, où la forme la plus répandue est uruga, mais on a des exemples aussi dans le galicien, dans le catalan, dans l'occitan et dans le sarde.

La partie centrale et orientale du domaine gascon, le département des Landes et une partie des départements adjacents, est occupé par le type gatamino, dans lequel on reconnaît le nom de la 'chatte', suivi par un nom, ou adjectif, mino, un terme caressant pour le chat utilisé aussi dans des autres aire de la Romania (par exemple à Gênes, comme je peux témoigner). On peut proposer la signification 'chatte-douce chatte' (comme le faisait Gaston Tuaillon). 
La dénomination 'chatte' de la chenille est bien répandue dans le domaine roman; il s'agit de dérivés du lat. CATTA. A côté des simples dérivés on trouve des syntagmes et des composés. Ce type se retrouve dans la partie septentrionale du domaine oïl (de la Bretagne à la Picardie) et dans l'Italie du nord-ouest, en Espagne on a seulement des points isolés. Les désignations signifiant 'chatte' sont presque toujours en contact avec le type '(petite) chienne', qui prédomine dans la langue d'oïl. Il est probable que certains noms, comme 'chatte' ou 'poilue' (ce dernier se trouve dans une vaste aire du domaine galloromain qui va de Boulogne à Nantes, mais est absent de l'aire gasconne), indiquent à l'origine surtout des insectes ayant des poils, tout à fait différents de la chenille verte sans poils. On a souligné ci-dessus que la carte 55 de l'Atlas de la Gascogne ne fait aucune différence entre les deux espèces.

Il est vraisemblable que l'aire de gatamino en Gascogne se soit enfoncée comme un coin dans le domaine primitif de ruca dont il ne reste aujourd'hui que deux tronçons. Il s'agit encore, je crois, d'un terme caressant pour se rendre favorable une petite bête qui peut être dangereuse.

Á ce propos, il faut noter que chaque animal est objet de croyances, qui peuvent être soit négatives soit positives : la chenille ne fait aucune exception, et au-delà des dommages qu'elle peut provoquer aux cultures on la considère dangereuse pour d'autres raisons, parfois très curieuses. Au point 699 SE de l'Atlas de la Gascogne on trouve le type 'taille-pieds', au point 692 SO 'tranche-doigts', et au point 687 NO un poda-nas 'tranche-nez' (accompagné par le type 'chenille'comme deuxième réponse, avec l'observation :"mot vieux ; aujourd'hui même les personnes âgées disent 2"). La chenille est accusée de maléfices qu'elle ne peut pas accomplir : en général le type 'tranche-doigt' ou similaires est un nom générique appliqué aux insectes pourvus de pinces. La chenille n'a aucune pince, mais est une bête très dangereuse pour les cultures. Son intentionnalité maléfique change dans la culture populaire, elle coupe les pieds, les nez des hommes, en catalan la chenille est appellée 'coupe-morve' : ce dernier nom n'est pas clair, mais la chenille est accusée encore de couper quelque chose aux êtres humains. En Engleterre on a recueilli la croyance qu'elle peut couper le pénis, ou bien le saigner !

Aux marges de l'aire gasconne on trouve le type chenille. Cette distribution est typique, et déjà observée par Gilliéron dans son commentaire à la carte "coq" de l'ALF. Comme on a vu ci-dessus pour le type 'chatte', la chenille est souvent nommée dans le domaine roman par des noms qui indiquent à l'origine d'autres animaux, parmi lesquels ceux qui désignent la 'chienne' ont une certaine diffusion. Il peut s'agir à la fois de mots désormais opaques ou des mots à la signification encore bien claire aux locuteurs.

Les dérivés de lat. CANICULA 'petite chienne' occupent la presque totalité de l'aire gallo-romane, à l'exception de l'extrême nord et du sud gascon et provençal. Cette grande extension n'a pas toujours sa cause dans l'influence de la langue standard : dans beaucoup de cas il s'agit de formes parallèles au fr. chenille plutôt que d'emprunts, mais deux aires occitanes, l'Ariège et le Gard, montrent des formes mal adaptées à la phonétique locale.

Le lat. CANICULA est une forme diminutive, pour laquelle la signification 'chenille' n'est pas attestée: en fait, ce mot pouvait signifier 'petite chienne', 'chien de mer' (un poisson), 'femme hargneuse', 'constellation du Chien', 'mauvais coup au jeu des dés'. Le glissement de sens n'est pas clair. On pourrait penser à une création déja latine, vue la grande diffusion de ce type dans le domaine galloroman. On ne parvient pas vraiment à imaginer une ressemblance extérieure entre chien et chenille, et du reste on trouve dans le domaine roman la chenille appellée 'lézard (vert)', 'grillon' ou 'chèvre'. Ce dernier nom est employé souvent aussi comme 
dénomination de la 'sauterelle' et du 'faucheur'.

On pourrait envisager dans cette dénomination une manifestation du tabouisme. Le tabou est une forme de magie négative, un moyen pour éviter un événement dangereux. Lanimal craint peut être appelé avec des noms caressants (cf. belette 'petite belle', ou l'équivalent it. donnola 'petite femme'), ou bien on peut éviter les possibles dangers (fictifs ou réels) si l'on n'appelle pas l'animal par son "vrai" nom, mais par des substituts (comme 'petite chienne' pour 'chenille').

Les désignations romanes de la coccinelle présentent le taux le plus élevé de variation lexicale parmi les animaux non domestiques. Partout, parmi les dénominations, on trouve des petites ou grandes bêtes (la poule et la vache, par exemple) et des noms de femmes. Cette diversité est due surtout à l'étroite liaison qui existe entre les croyances populaires à propos de la coccinelle et de ses dénominations. La coccinelle est souvent représentée clairement comme un intermédiaire entre les hommes (surtout les enfants aujourd'hui) et le divin : les dénominations positives sont poussées au plus haut niveau, le ciel, Dieu, la Vierge, les Saints, les gens d'église, les rois. Une grande partie des noms romans de la coccinelle se laisse expliquer à la lumière des formulettes enfantines (parfois de véritables exorcismes) auxquels ils sont liés. Dans les langues romanes, un des motifs les plus communs dans ces formulettes, «l'invitation à voler», devient une des plus fréquentes dénominations de notre insecte : oïl Paris-vole, Marivole, occitan boloPaoulo, portugais joaninha voa, italien Mariavola, vola tout-court etc.

La carte 1223 "coccinelle" de l' Atlas Linguistique et ethnographique de la Gascogne ne répresente pas une exception. Les auteurs ont receuilli un grand nombre de formulettes par lesquelles les enfants jouent à provoquer l'envol de la coccinelle, formulettes qui ne sont pas reproduites dans la carte. Lapostrophe initiale de la formulette "vole, X" devient un substantif, dont le genre grammatical dépend de celui du substantif: la bolo Mario sera alors féminin.

Les auteurs ont superposé à la carte 1223 des noms de la coccinelle une indication générale sur la diffusion de ces apostrophes (bolo Mario, bolo Pau etc.). Le nom de personne le plus fréquent en Gascogne pour appeler la coccinelle est Marie, qui est le nom féminin le plus fréquemment utilisé dans les langues romanes : la référence est à la Vierge Marie, la mère de Dieu. Il a cependant subi un procès de désacralisation qui l'a rendu plus familier, mais au point 692 S on trouve la forme encore sacralisée Sainte Marie. Il est souvent accompagné partout de diminutifs ou de l'injonction «vole !». En Gascogne, dans les formes enregistrées par l'Atlas, on trouve soit Vole Marie que Marie vole. Parmi les autres noms de personne, on trouve Paul, forme qui est limitée à l'Ariège. Les noms masculins sont relativement rares comme noms de la coccinelle dans la Romania. Le prénom masculin le plus fréquent lié à la coccinelle est le type signifiant 'Saint Antoine' très répandu en Espagne. On trouve aussi, dans quelques points de la Gascogne, le nom masculin Giraut comme nom de la coccinelle, dans les formules déjà vues pour le type Marie : vole Giraut, Saint Giraut, vole Saint Giraut.

Quant au type pivole, pibole 'coccinelle', qui occupe la partie nord-orientale de la carte 1223, son explication reste pour moi douteuse : la deuxième partie du mot peut être liée aux types Marie vole etc. discutés ci-dessus, mais la première partie n'est pas claire: on pourrait la rattacher au nom de la pie, mais la pie est désignée dans cette aire par le nom ajasse. On pourrait penser alors à une connection avec des mot comme pirouette. Pibole est aussi le nom de la toupie en Anjou (au nord de la zone concernée).

Quant aux noms de personne, on trouve encore, au point 658 NO, le nom Marguerite, et au point 657 Madeleine. Le nom Catherine se trouve dans un point des Bas Pyrénées. Il s'agit 
d'un nom moins banal que Marie. Comme dénomination simple de la coccinelle Catherine se trouve au Portugal, en Espagne, en France, Belgique et Italie. À Santander les enfants lui chantent : «Catalina, lina, lina/ sube al cielo y dile a Dios/ que mañana haga mucho sol» («Catherine monte au ciel et dis à Dieu que demain il fait grand soleil»).

Jeanne Marie ou Marie Jeanne sont relevés dans deux points des Hautes-Pyrénées et dans le Gers. Le type Jean ou Jeanne est fréquent dans les langues romanes comme dénomination de la coccinelle. Au Portugal, le nom le plus fréquent de la coccinelle est joaninha.

Au point 641, dans la Gironde, les coccinelles sont appelées soldats. Les noms de métier sont donnés, en petit pourcentage, à toutes les bestioles étudiées par l'Atlas Linguistique Roman. Il nous paraît inutile de chercher une motivation particulière pour ces appellations. La coccinelle ne fait pas exception à la règle. Le nom de 'soldat' pour la coccinelle se trouve aussi dans quelque point d'Italie.

Autres dénominations de la coccinelle en Gascogne : les types 'bête', ou des termes génériques pour 'insecte' comme barbaroto, barbot, kuko. Dans l'utilisation d'un générique tel que 'animal' ou 'petite bête' transparaît, comme dans beaucoup d'autres cas, un refus de nomination qui souligne que le vrai nom de la coccinelle ne doit pas être prononcé, même si celle-ci doit être nommée pour obéir aux questions qu'on lui pose.

\section{Références}

Caprini, Rita. 1989. Ancora sui nomi della coccinella septempunctata. Quaderni di Semantica X(1). 5-11.

Caprini, Rita. 1999. Tre saggi sul bruco: presentazione. Quaderni di Semantica XX(2). 197-198.

Caprini, Rita. 1999b. I nomi del bruco in area romanza: rileggendo il 'bruco' di Richard Riegler. Quaderni di Semantica XX(2). 209-223.

Caprini, Rita. 2001. Zoonimi popolari romanzi: il caso del bruco. In La dialettologia oggi fra tradizione e nuove metodologie, Atti del Convegno Internazionale, Pisa 10-12 febbraio 2000, a cura di A. Zamboni, P. Del Puente, M.T. Vigolo, Pisa: Edizioni ETS. 283-294.

Caprini, Rita. 2001b. Chenille, carte et Commentaire. In Atlas Linguistique Roman, vol. II/a, pp. 61-87, carta non numerata. Roma: Istituto Poligrafico e Zecca dello Stato.

Caprini, Rita. 2004. La motivazione nella zoonimia. In Etnolinguistica e zoonimia, a cura di Antonio Mendicino, Nadia Prantera, Marta Maddalon, Rende: Università degli Studi della Calabria. 25-32.

Caprini, Rita. 2005. Meaning, semantics, taboo, onomasiology and etymology. In Atti del Convegno "Animal Names", Venezia, 2-4 ottobre 2003, a cura di Alessandro Minelli, Gherardo Ortalli, Glauco Sanga. Venezia: Istituto Veneto di Scienze Lettere e Arti. 235244.

Caprini, Rita. 2005b. Nomi romanzi del pettirosso. Quaderni di Semantica XXVI(1). 77-107.

Caprini, Rita. 2009. Les désignations romanes du rouge-gorge. In Atlas Linguistique Roman, vol. IIb. Roma: Istituto Poligrafico e Zecca dello Stato. 425-459.

Caprini, Rita. (in stampa). Coccinelle, Carte et Commentaire. In Atlas Linguistique Roman. Roma: Istituto Poligrafico e Zecca dello Stato.

Séguy, Jean. 1954. Atlas Linguistique et ethnographique de la Gascogne. Paris: CNRS. 